\title{
Autonomous Pick-and-Place of Pneumatically Attachable Flexible Rails
}

\author{
C. D‘Ettorre ${ }^{1}$, A. Stilli ${ }^{1}$, G. Dwyer ${ }^{1}$, M. Tran ${ }^{2}$ and D. Stoyanov ${ }^{1}$ \\ ${ }^{1}$ Wellcome/ EPSRC Centre for interventional and Surgical Science (WEISS), \\ ${ }^{2}$ Research Department of Surgical Biotechnology, University College London \\ c.dettorre@ucl.ac.uk
}

\section{INTRODUCTION}

In Robotic-Assisted Partial Nephrectomy (RAPN) tumours are excised from the kidney while aiming to preserve organ functionality. Ultrasound (US) imagining is used as an intraoperative technique to identify and outline of the tumour. During RAPN with the da Vinci Surgical System (Sunnyvale, CA), US images are acquired using a "drop-in" US probe which is passed through the instrument port. The EndoWrist ${ }^{\circledR}$ Pro-Grasp Forceps Gripper is commonly used to grasp this kind of probe and to swipe it on the surface of the kidney. During scanning, given the morphology of the kidney, slippages are common, and repositioning of the probe is often needed.

In this paper, we build on previous work [1] where Pneumatically Attachable Flexible (PAF) Rails were introduced. We propose a software architecture customized for PAF to automate track-guided US scanning leading towards better workflow. Our architecture is designed to automatically grasp the PAF rail and to place it on the tissue. After autonomous pickand-place of the system, the probe can be used for scanning, sliding the probe along the PAF rail. The architecture relies on a calibrated environment and on vision as input of a gradient-based planning algorithm.

\section{MATERIALS AND METHODS}

Calibration and Visual Feedback - Calibration is needed to link the vision system and measurements from it with robot kinematics data. The stereo endoscope is calibrated using conventional camera calibration [2].

For the transformation between the camera and robot frame, we select a specific point cloud and, knowing the coordinate of points in the different reference frames, the calibration problem can be formulated as a point cloud registration problem [3].

The proposed system presents a visual interface which allows the surgeon to select the current position of the PAF rail and the location where it should be placed on the organ's surface. Selection of the grasping site represents the input to the system. The coordinates of the selected point are then triangulated and reconstructed in the 3D space using the stereo endoscope. They can then be used as targets for the pick and place task using the calibration transformations. In parallel, the kidney is segmented both in the left and right frame and the local features are extracted using the SURF algorithm [4] for both frames. The two points clouds are triangulated, reconstructed and translated in

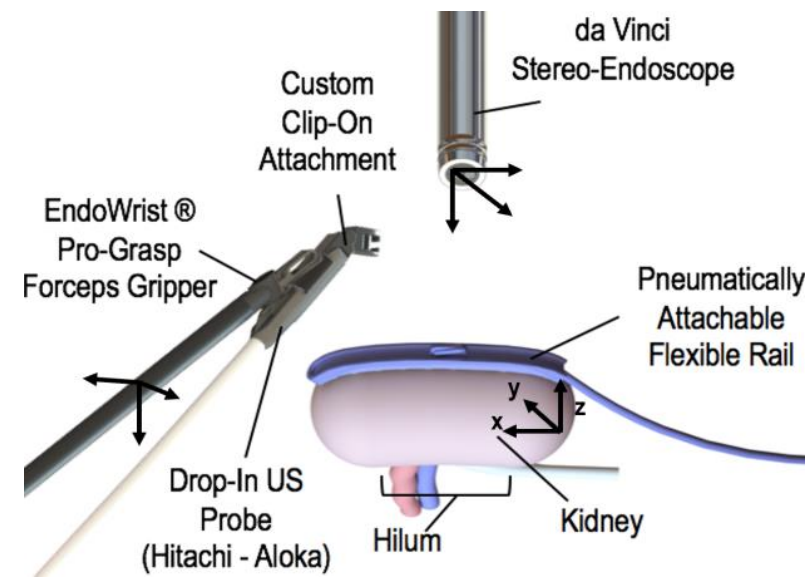

Fig. 1 Schematic representation of the proposed system. The PAF rail is located on the kidney surface and the model of US-probe is equipped with an adaptor. This allows the surgeon to properly grasp the probe with the Pro-Grasp tool and slide the probe in the rail.

the robot reference frame and used as the target for the placement phase.

Gradient-based Planning - Our algorithm takes as input the current and desired positions of the PAF rail defined by the surgeon and allows the robot to grasp it and position it on the kidney's surface accordingly. Notably, we have not considered the effects of physiological motion on the organ. However, in consideration of the future work we will conduct in this direction, we have decided to choose a fast planning algorithm to allow rapid re-planning, hence, to tackle the position uncertainty associated with motion in vivo.

At each time step, given the current location of the tooltip in the Cartesian space, we computed an intermediate position, through the gradient-based approach, located at a distance called next-translation. If the target is within the next-translation distance, the new Cartesian position is the target itself. Similarly, for the orientation, we compute an intermediate target as the rotation necessary to change the current orientation of the tooltip to the target one. Given the morphology of the system paired with the organ we defined as the target orientation the one along the $\mathrm{x}$-axis (direction shown in Fig. 1). The intermediate rotation, the next-rotation, is parameterized as the angular rotation that needs to be performed to reach the target orientation. Due to the design of the grasping slot of the PAF rail, it is important to correctly grasp the system with the tooltip in the proper orientation, to guarantee an effective grasp. Hence, after the grasp-acquisition step, 
the orientation is never more than a small angle away from the desire targeted organ surface orientation. We assume that the orientation of the PAF rail in the operating area before the grasp can be controlled by the surgeon's assistance through the pressure pipe. Using cisst-saw library [5], we compute the Cartesian Jacobian and the rotational Jacobian, which show how the changes in the joint angles effect the final pose of the tooltip in the Cartesian space. Rotations are given in angle-axis representation. The parameters next-transl and next-rot, associated with the next-translation distance and the next-rotation angles respectively, guarantee that the intermediate tool tip pose is close to the current one. Hence, it is possible to linearize our problem using Equation 1, where $\mathbf{J}_{\text {tr }}$ and $\mathbf{J}_{\mathbf{r}}$ are the Cartesian and rotational Jacobian defined above, and $\boldsymbol{\Delta}_{\mathbf{t r}}$ and $\Delta_{\mathbf{r}}$ the desired translation and rotation to move the end effector to the intermediate target. The da Vinci robotic arm has 6 DoFs and an ordinary least-squares regression is used to compute the required joint changes necessary to satisfy the equation.

$$
\left[\begin{array}{c}
\boldsymbol{J}_{t r} \\
\boldsymbol{J}_{r}
\end{array}\right] \boldsymbol{\theta}=\left[\begin{array}{c}
\Delta_{t r} \\
\Delta_{r}
\end{array}\right]
$$

\section{RESULTS}

A dataset of 40 acquisitions has been recorded to test the overall architecture. To recreate the surgical scenario, the experiment was defined as follows (see Fig. 2): 1 - the PAF rail is randomly inserted inside the field of view of the stereo-endoscope; 2 - the operator triggers the motion of the robot selecting on screen the desired position of the PAF rail on the targeted kidney; 3 - The robot grasps the PAF rail; 4 - it moves toward the target organ; 5 - The robot place the PAF rail on the organ surface according to the position previously selected through the interface. The task in consider successfully completed once the PAF rail is in suction and is correctly positioned on the kidney's surface. The experiment had a success rate of 35 acquisitions over 40 (Fig. 3), while in the remaining 5/40 the tooltip was not able to reach the rail to complete the grasp. The average completion time among all the successful acquisitions was 42 seconds.

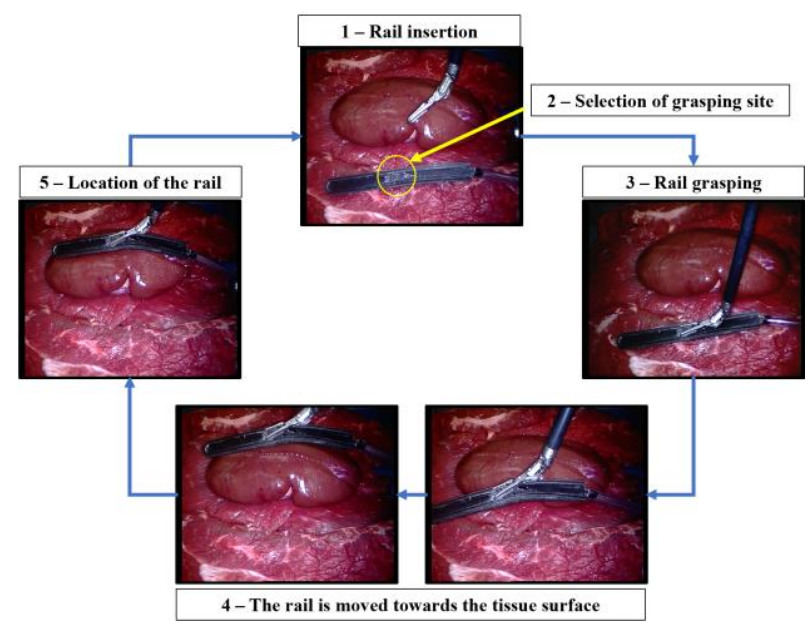

Fig. 2 Representation of the different step of the pick and place task.

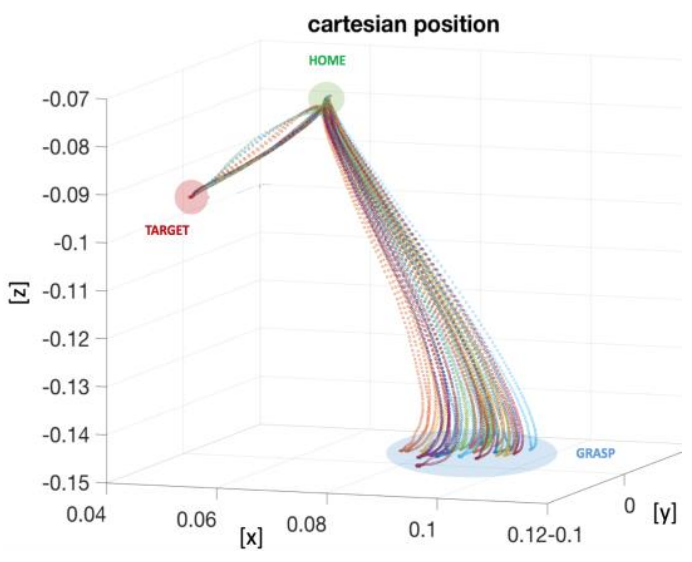

Fig. 3 Representation of the Cartesian position of the tooltip in the workspace in meters. The 35 successful acquisitions are represented.

\section{DISCUSSION}

The experiments presented in this paper show that the autonomous pick-and-place of the PAF rail system is feasible. We showed that the proposed software architecture can be successfully used to locate the PAF rail in the environment, to place it in a desired position, as well as to move the robotic instruments accordingly based on the calibrated environment and on a gradientbased trajectory planned on the fly. The results obtained show a success rate of $87.5 \%$. Regarding the rest of executions, the system fails the correct execution for reasons related to the accuracy of the reconstruction of the grasping site. As a matter of fact, the small baseline between the two cameras of the stereo endoscope makes really challenging to precisely estimate of the $3 \mathrm{D}$ position of points in space. Several challenges remain: one is increasing and incorporating mitigation strategies for the uncertainties coming from the real clinical environment, e.g. kidney motion and deformation and a highly vascularized environment where bleeding can cause occlusion, thus the failure of the vision system. This could have potentially adverse effects also on the surface registration process.

\section{REFERENCES}

[1] A. Stilli et all, "Pneumatically Attachable Flexible Rail for Track-Guided Ultrasound Scanning in RoboticAssited Partial Nephrectomy. A Preliminary Design Study." IEEE Robotics And Automation Letters, Vol. 4, no. 2, pp. 1208-1215, 2019.

[2] Z. Zhang, "A Flexible New Technique of Camera Calibration.", IEEE Translation of Pattern Analysis And Machine Intelligence, vol. 22, pp. 1330-1334, 2000.

[3] C. D'Ettorre et all, "Automated Pick-up of a Suturing Needle for Robotic Surgical Assistance", ICRA, pp. 1370-1377, 2018.

[4] H. Bay et all, "SURF: Speeded up Robust Features", 2006.

[5] P. Kazanzides et all, "An Open Source Research Kit for the da Vinci Surgical System", in ICRA, 2014, pp. 6434 6439 . 